\title{
1 Measuring hearing in wild beluga whales
}

2

3 T. Aran Mooney ${ }^{1 *}$, Manuel Castellote ${ }^{2,3^{*}}$, Lori Quakenbush ${ }^{4}$, Roderick Hobbs ${ }^{2}$, Caroline 4 Goertz $^{5}$, Eric Gaglione ${ }^{6}$

5

$6{ }^{1}$ Biology Department, Woods Hole Oceanographic Institution, Woods Hole, MA 02543, 7 USA

$8 \quad{ }^{2}$ National Marine Mammal Laboratory, Alaska Fisheries Science Center, National Marine

9 Fisheries Service, Seattle, WA 98115

$10{ }^{3}$ North Gulf Oceanic Society, Homer, AK 99603, USA.

$11{ }^{4}$ Alaska Department of Fish and Game, 1300 College Road, Fairbanks, AK. 99701, USA

$12 \quad{ }^{5}$ Alaska SeaLife Center, Seward, AK 99664, USA

$13{ }^{6}$ Georgia Aquarium, 225 Baker St NW, Atlanta, GA 30313, USA

14

15 *These authors contributed equally to this work

16

17 Emails: amooney@whoi.edu, manuel.castellote@noaa.gov, lori.quakenbush@alaska.gov,

18 rod.hobbs@noaa.gov, egaglione@georgiaaquarium.org

19

20 Corresponding author: T. Aran Mooney, WHOI, 266 Woods Hole Rd, MRF MS\# 50, Woods

21 Hole, MA 02543, USA; amooney@whoi.edu

22

23 Key words: anthropogenic noise, sensory, marine mammal, cetacean, odontocete, arctic 


\section{Abstract}

2 We measured the hearing abilities of seven wild beluga whales (Delphinapterus leucas)

3 during a collection-and-release experiment in Bristol Bay, AK, USA. Here we summarize the

4 methods and initial data from one animal, discussing the implications of this experiment.

5 Audiograms were collected from 4-150 kHz. The animal with the lowest threshold heard best

6 at $80 \mathrm{kHz}$ and demonstrated overall good hearing from 22-110 kHz. The robustness of the

7 methodology and data suggest AEP audiograms can be incorporated into future collection-

8 and-release health assessments. Such methods may provide high-quality results for multiple

9 animals facilitating population-level audiograms and hearing measures in new species.

\section{1. Introduction}

11 Hearing is the primary sensory modality for odontocete marine mammals. They are

12 generally considered to have sensitive hearing and may detect a broad range of frequencies.

13 Relying on hearing can be particularly adaptive in the marine environment where light and

14 other cues are often limited and natural sounds are frequently abundant. Yet, these sensitive

15 auditory abilities may also be easily impacted by anthropogenic noise.

16 Human use of the Earth's oceans has steadily increased over the last century resulting in

17 an increase in anthropogenically produced noise (e.g., National Academy of Sciences 2003).

18 The Arctic is no exception to this increase (Blackwell \& Greene 2003). Reductions of polar sea

19 ice and the opening of the Northwest Passage presumably will open up habitat for many top

20 predators. Yet, this decrease in sea ice provides greater human access to high latitude

21 environment and such a change poised to transform a relatively pristine environment into to

22 one saturated with human activities and associated noise. Sources are varied and include: naval

23 exercises, boundary definitions, shipping/movement along Alaska's North Slope, seismic

24 resources exploration, and the construction of infrastructure needed to support it (Wang \&

25 Overland 2009; Titley \& St. John 2010). These changes encompass habitats of Delphinapterus 
1 leucas (beluga whales) and other top predators. Despite this obvious overlap of human-natural

2 interests, there is a poor understanding of influences of these sound-associated changes. In

3 order to estimate the impacts of this noise it is crucial to evaluate the natural hearing abilities

4 and the variation with marine mammal populations.

5 Yet a primary challenge is that audiograms of odontocetes marine mammals have most

6 often been estimated from stranded animals or non-wild individuals (for a review see Mooney

7 et al. 2012). In many instances, these records have produced valuable data that is otherwise

8 unavailable. For example, hearing in several stranded beaked whale species have helped define

9 what these sound-sensitive animals hear (Finneran et al. 2009; Pacini et al. 2011). The

10 audiogram of a stranded infant Risso's dolphin helped redefine what the species actually

11 detects (Nachtigall et al. 2005). Work with trained odontocetes provides scientific data that is

12 likely unique to those settings and can address how animals hear or how they may be protected

13 from anthropogenic noise (Nachtigall \& Supin 2008). Yet, in many instances health

14 compromised, stranded animals may not have normal auditory abilities, thus not necessarily

15 representative of wild populations. Further, without baselines to wild individuals it is difficult

16 to put differences and results of non-wild individuals in a relative context. Clearly, there is

17 value in increasing the number of animals within a species measured for hearing capabilities

18 whenever possible.

19 Here we describe methods and initial results for the measuring the hearing of wild $D$.

20 leucas (Castellote et al. 2013). The goal of this study was to determine hearing sensitivity in

21 wild Bristol Bay D. leucas, during a planned collection-and-release operation. Monitoring of

22 D. leucas has been recommended in recent years because this species is likely to be

23 negatively impacted by climate change, but also because such a broadly dispersed, high

24 trophic feeder can serve as an effective sentinel of the ecosystem(s) in which they live 
1 (Moore 2008; Moore \& Huntington 2008; Simpkins et al. 2009). Because noise may impact

2 D. leucas in a variety of ways, it is essential to determine what these animals hear.

3 In view of the expected changes in the Arctic acoustic environment, expanding our

4 knowledge on D. leucas hearing is of central importance for an appropriate conservation

5 management framework. One of the five distinct stocks of D. leucas whales that are currently

6 recognized in U.S. waters, the Cook Inlet D. leucas population, is endangered and efforts for

7 its recovery to date have not been successful. The impact of anthropogenic noise has been

8 identified as a serious threat potentially impeding its recovery (NMFS 2008). On the

9 contrary, the Bristol Bay D. leucas population is increasing and is considered to be a healthy

10 population (NMFS 2008). The acoustic environment in Bristol Bay is different; many of the

11 chronic anthropogenic sources typically found in Cook Inlet D. leucas habitat are essentially

12 absent or seasonally present at lower intensities in Bristol Bay habitat. This suggests that

13 Bristol Bay D. leucas are a valuable asset to evaluate baseline hearing and health measures

14 for comparison to effected populations, such as Cook Inlet D. leucas.

16 2. Temporary collection of beluga whales and hearing tests methods

17 This study was conducted in September, 2012 in Bristol Bay AK, USA. The

18 audiograms were measured during an overall health assessment study that required the

19 collection-and-release of D. leucas. Audiograms were obtained on seven of seven belugas

20 tested. The procedures were similar to those followed by (Ferrero et al. 2000) and were

21 conducted under National Marine Fisheries Service marine mammal research permit \#14245

22 and approved by the necessary Institutional Animal Care and Use Committees. The full

23 results are to be published elsewhere (Castellote et al. 2013); here we provide a summary of

24 the methods and preliminary results. 
1 Bristol Bay is a generally shallow, muddy-bottomed estuary system that supports a

2 population of $D$. leucas. Using three $3.5 \mathrm{~m}$ aluminum skiffs and one soft-bodied inflatable

3 boat, we would search for an adult beluga. When a suitable animal was spotted (Fig. 1), one

4 of the skiffs would follow and gradually approach the whale to encourage it to swim into

5 shallow water $(<2 \mathrm{~m})$. From one of the boats, a $125 \mathrm{~m}$ long by $4 \mathrm{~m}$ deep net, of $0.3 \mathrm{~m}$

6 braided square mesh, was deployed around the whale. Once the deployment boat and net

7 encircled the whale the inflatable boat approached the outside of the net and three handlers

8 placed a soft tail-rope around the whale's peduncle. The rope's other end was fixed to the

9 inflatable boat to secure the whale. The large net was gradually recalled while a "belly-

10 band" stretcher was placed under the D. leucas. Handholds in this stretcher facilitated

11 adjusting the whale's position as the water depth changed with the tide. The animal was then

12 positioned parallel to the small inflatable boat. The $D$. leucas's head was typically rested on

13 or just above the soft mud bottom, keeping the lower jaw and primary hearing pathways

14 below the water surface. The animal's blowhole was generally above the surface. This setup

15 was consistent for all animals, except one for which the water level was too low and this test

16 was conducted partly out of the water. Animals were maintained in this position for the

17 audiogram and health exam. The AEP test equipment was outfitted in a ruggedized case; both

18 it and the operator sat in the small inflatable boat beside the $D$. leucas during the hearing tests

19 (Fig. 1).

\section{3. Discussion of results}

22 Audiograms were successfully collected on all seven adult D. leucas whales

23 temporarily collected and tested. Evoked response waveforms and envelope following

24 responses were generally easily identifiable and distinct from the background

25 electrophysiological noise. The inset in Figure 2 shows an EFR that was recorded using 
1 stimuli ca. $20 \mathrm{~dB}$ about the hearing threshold at $32 \mathrm{kHz}$. Such a measurement would take ca.

$230 \mathrm{sec}$ to collect. Thus, overall thresholds at a particular frequency were obtained in 3-5 min.

3 This relatively rapid threshold measurement facilitated collecting multiple thresholds per

4 animal but also minimizing the “with-animal” time. For example, animal \#7’s audiogram

5 consisted of 12 frequencies tested. Two of these (4 and $150 \mathrm{kHz}$ ) did not induce measureable

6 AEPs. The entire data set was collected in 55 min which includes multiple breaks for other

7 measurements such as blood samples or repositioning the animal. Records were collected in

8 concert with a suite of other measurements with no discernible impact on the physiological

9 noise. This allowed for a relatively efficient of data collection when compared to behavioral

10 methods which require significant time to train animals and conduct experiments. It is also

11 relatively quick for other AEP audiograms which make take multiple days (sessions). Here

12 we collected seven audiograms over six field days (including one day which was poor

13 weather conditions and no whales were sighted).

14 Despite the potential challenges of the experiment (cold conditions, electrophysiology

15 close to the water, confined spaces, concurrent measurements potentially introducing noise,

16 safety and welfare of the people and animals) the audiograms were of very good quality.

17 They are of equal quality to the field-based collection-release audiometric data of Cook and

18 Mann (2004) for bottlenosed dolphins (Tursiops truncates) and of Nachtigall et al., (2008;

19 see also Mooney et al. 2009) for white-beaked dolphins (Lagenorhynchus albirostris) Our

20 success both in ease and safety of data acquisition and quality of the data suggest the methods

21 could easily be applied to other species in similar situations. This is of particular importance

22 for populations where anthropogenic noise is chronic and has been identified as a potential

23 stressor. Examples are the endangered Cook Inlet D. leucas or the threatened St. Lawrence D.

24 leucas populations. The prevalence of anthropogenic noise in their habitat and its cumulative

25 effects might be compromising the survival of both D. leucas populations (NMFS 2008; 
1 DFO 2012). This assertion is based on current knowledge of the level and acuity of

2 anthropogenic noise in these ecosystems (e.g., Gervaise et al. 2012) and our understanding on

3 D. leucas hearing and acoustic communication. However, because of the inherent difficulties

4 in evaluating noise impact on cetaceans, there are no data supporting this hypothesis.

5 Audiograms using the method described here could be collected in Cook Inlet and in the St.

6 Lawrence Estuary to measure the hearing of $D$. leucas with greater exposed to anthropogenic

7 noise and be compared to the baseline audiogram described for Bristol Bay D. leucas.

\section{Acknowledgements}

10 Project funding and field support provided by Georgia Aquarium and the National Marine

11 Mammal Laboratory of the Alaska Fisheries Science Center (NMML/AFSC). Field work also

12 supported by National Marine Fisheries Service Alaska Regional Office (NMFS AKR),

13 WHOI Arctic Research Initiative, WHOI Ocean Life Institute, U.S. Fish and Wildlife

14 Service, Bristol Bay Native Association, Alaska SeaLife Center, Shedd Aquarium and Mystic

15 Aquarium. Audiogram analyses were funded by the Office of Naval Research award number

16 N000141210203 (from Michael Weise). We also acknowledge substantial assistance

17 R.Andrews, G. Biedenbach, B. Long, S. Norman, M. Keogh, A. Moors, L. Thompson, T.

18 Binder, L. Naples, L. Cornick, K. Royer, and K. Burek-Huntington, R. Hiratsuka, A. Roehl,

19 B. Tinker and D. Togiak. All work conducted under NMFS permit no. 14245 and in

20 accordance with approval from the NMML/AFSC IACUC protocols (ID number: AFSC-

21 NWFSC2012-1) and WHOI IACUC protocols (ID number: BI166330).

\section{References}

24 Castellote $\mathrm{M}$ et al. (2013) Baseline hearing abilities and variability in wild beluga whales

25 (Delphinapterus leucas). J Exp Biol Submitted 
1 Cook MLH, Mann DA (2004) Auditory brainstem response hearing measurements in

2 free-ranging bottlenose dolphins (Tursiops truncatus). J Acoust Soc Am 116:2504.

3 DFO (2012) Recovery strategy for the beluga whale (Delphinapterus leucas) St.

4 Lawrence estuary population in Canada. Species at Risk ActRecovery Strategy Series.

5 DFO, Ottawa

6 Ferrero RC, Moore SE, Hobbs R (2000) Development of beluga, Delphinapterus leucas,

7 capture and satellite tagging protocol in Cook Inlet, Alaska. Marine Fisheries Review

$8 \quad 62: 112-123$.

9 Finneran JJ, Houser DS, Mase-Guthrie B, Ewing RY, Lingenfelser RG (2009) Auditory

10 evoked potentials in a stranded Gervais' beaked whale

11 (Mesoplodon europaeus). J Acoust Soc Am 126:484-490.DOI: 10.1121/1.3133241

12 Gervaise C, Roy N, Kinda B, Menard N (2012) Shipping noise in whale habitat:

13 Characteristics, sources, budget, and impact on belugas in Saguenay-St. Lawrence

14 Marine Park hub. J Acoust Soc Am 132:76-89.

15 Mooney TA, Nachtigall PE, Taylor KA, Miller LA, Rasmussen M (2009) Comparative

16 auditory temporal resolution of the white-beaked dolphin (Lagenorhynchus albirostris).

17 J Comp Physiol A 195:375-384.

18 Mooney TA, Yamato M, Branstetter BK (2012) Hearing in cetaceans: From natural

19 history to experimental biology. Advances in Marine Biology 63:197-246.

20 Moore SE (2008) Marine mammals as ecosystem sentinels. Journal of Mammology

21 89:534-540.

22 Moore SE, Huntington HP (2008) Arctic marine mammals and climate change: impacts

23 and resilience. Ecological Applications 18:S157-S165.

24 Nachtigall PE et al. (2008) Shipboard measurements of the hearing of the white-beaked

25 dolphin, Lagenorynchus albirostris. J Exp Biol 211:642-647. 
1 Nachtigall PE, Mooney TA, Taylor KA, Yuen MML (2007) Hearing and auditory evoked

2 potential methods applied to odontocete cetaceans. Aquat Mamm 33:6-

$3 \quad$ 13.doi:10.1578/AM.33.1.2007.6

$4 \quad$ Nachtigall PE, Supin AY (2008) A false killer whale adjusts its hearing when it

5 echolocates J Exp Biol 211:1714-1718.doi:10.1242/jeb.013862

6 Nachtigall PE, Yuen MML, Mooney TA, Taylor KA (2005) Hearing measurements from a

7 stranded infant Risso's dolphin, Grampus griseus. J Exp Biol 208:4181-4188.

8 National Academy of Sciences (2003) Ocean noise and marine mammals. National

9 Academies Press, Washington, DC

10 NMFS (2008) Conservation Plan for the Cook Inlet beluga whale (Delphinapterus

11 leucas). National Marine Fisheries Service, Juneau, Alaska

12 Pacini AF et al. (2011) Audiogram of a stranded Blainville's beaked whale (Mesoplodon

13 densirostris) measured using auditory evoked potentials. J Exp Biol 214:2409-

$14 \quad$ 2415.doi:10.1242/jeb.054338

15 Simpkins M, Kovacs KM, Laidre K, Lowry L (2009) A framework for monitoring arctic

16 marine mammals. Conservation of Arctic Flora and Fauna, Circumpolar Biodiversity

17 Monitoring Programme Report No. 16:pp 12.

18 Titley D, St. John C (2010) Arctic security considerations and the U.S. Navy's Roadmap

19 for the Arctic. Naval War College Review 63:35-48.

20 Wang M, Overland J (2009) A sea ice fre Arctic within 30 years. Geophysical Research

21 Letters 36:L07502.

\section{Figure captions}

24 Figure 1. (A) Spotting a D. leucas from the aluminum skiff. (B) The AEP audiogram setup. 25 The recording, reference and ground electrode are noted with from posterior to anterior (right 
1 to left) by the yellow arrows. A measure of breath is also being taken concurrently. (C) The

2 AEP system in its case and (D) in the soft inflatable boat during data recording.

3

4 Figure 2. The AEP audiogram and waveform (inset) of D. leucas \#7. This animal had the 5 overall mean lowest threshold.

6

7 
Figure 1
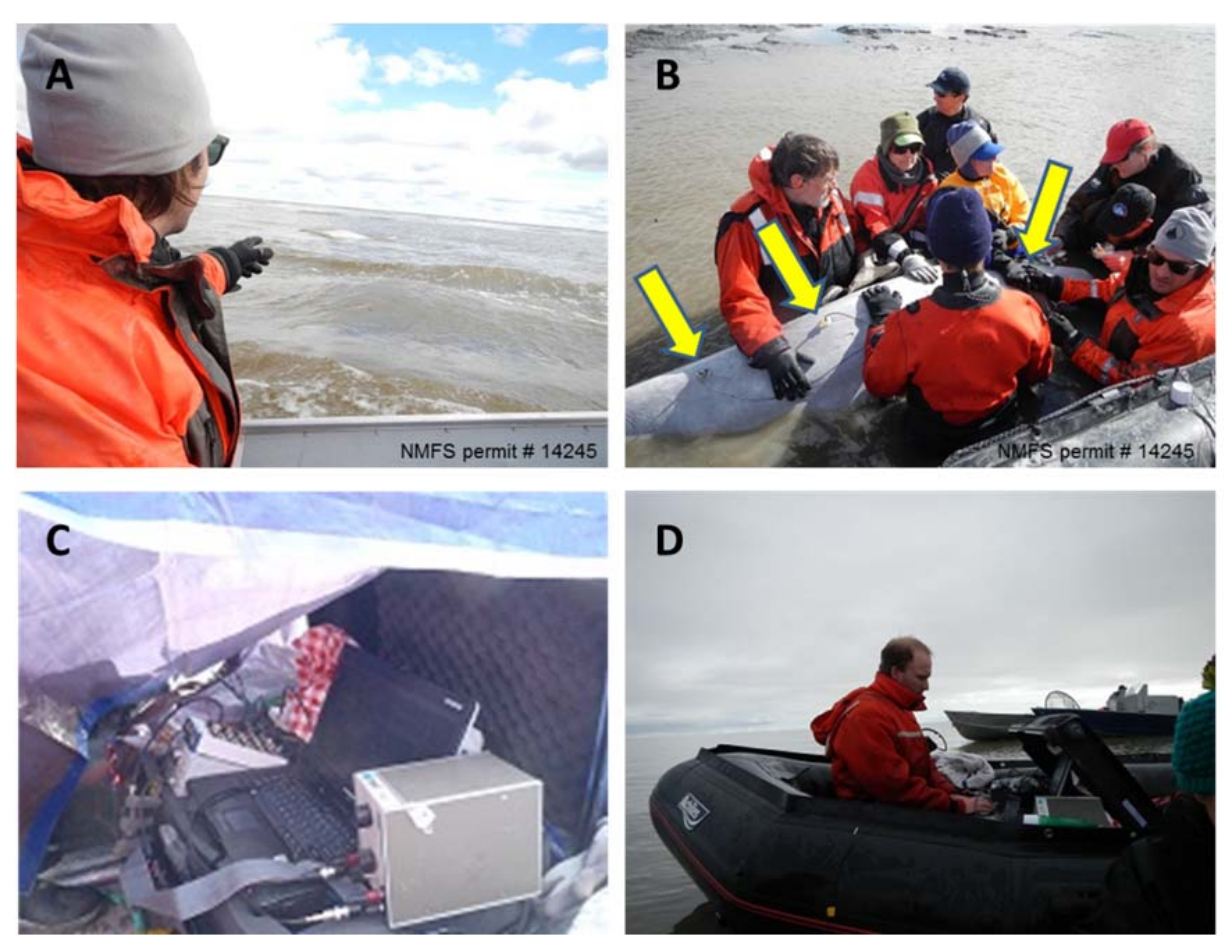
Figure 2.

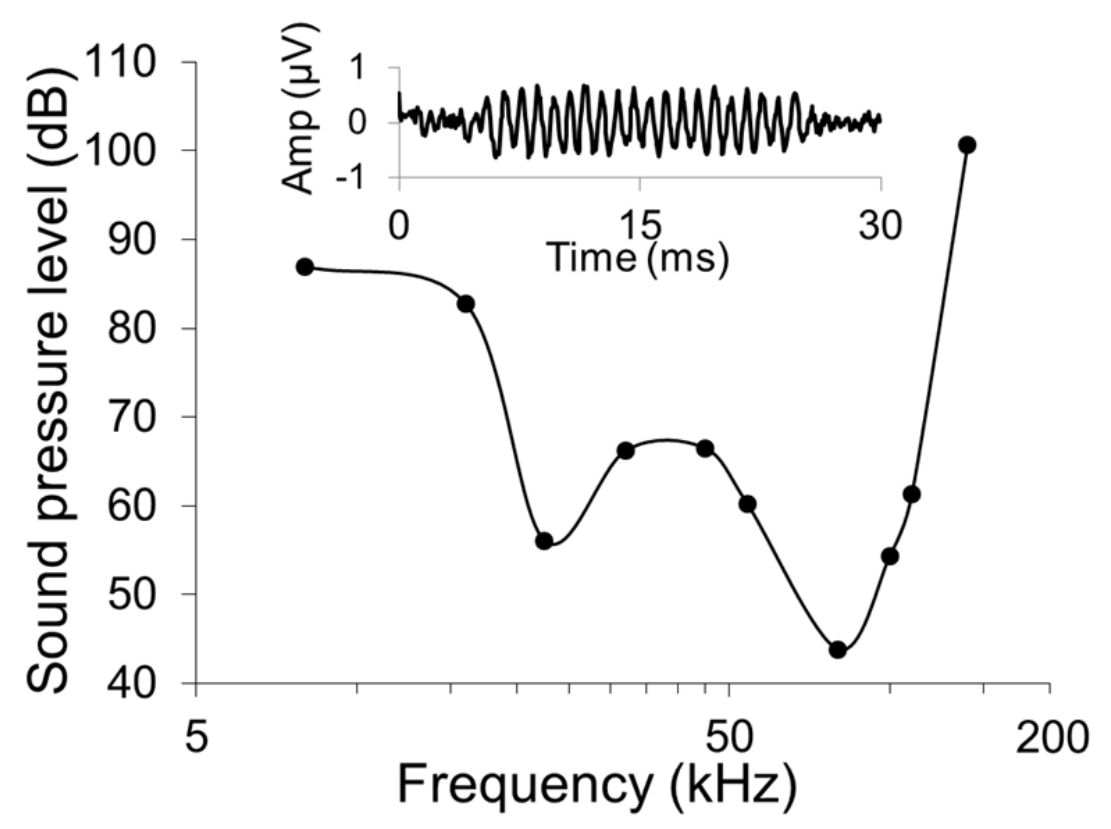

